\title{
Fine-grained Data Usage Analysis by Access Sampling
}

\author{
Seeing The Data That is Not There
}

\author{
Zhizhou Zhang* \\ University of California, Santa \\ Barbara \\ Santa Barbara, California \\ zhizhouzhang@ucsb.edu
}

\author{
Chencheng Ye \\ Huazhong University of Science and \\ Technology \\ Wuhan, China \\ yechencheng@gmail.com
}

\author{
Rahman Lavaee* \\ Google Inc. \\ Sunnyvale, California \\ rahmanl@google.com
}

\author{
Ning $\mathrm{Gu}^{*}$ \\ Rutgers University \\ Piscataway, New Jersey \\ ning.gu@rutgers.edu
}

\author{
Chen Ding \\ University of Rochester \\ Rochester, New York \\ cding@cs.rochester.edu
}

\begin{abstract}
Estimating active data usage is a basic problem in memory system analysis, management and optimization. Finegrained usage analysis is costly because it requires monitoring data access.

This paper presents efficient fine-grained analysis through access sampling. By taking random samples at some frequency ratio, e.g. $1 \%$ of cache misses, it infers the size of the other data accessed in the rest of the trace. Since the analysis deduces the total amount of data accessed by inspecting a subset of accesses, it is seeing the data that is not there. The paper presents the analysis and its evaluation using 8 program traces. The error of data-size prediction is $33 \%$ at $1 \%$ sampling and $6 \%$ at $10 \%$ sampling. The new technique is significantly more accurate than two previous models. One is based on skewed distributions, i.e. the " $80-20$ " law. The other is the well-known Good-Turing frequency estimation.
\end{abstract}

\section{Introduction}

On modern computer systems, memory has become often the largest factor in cost, power and energy consumption. Memory is also increasingly diverse, with different materials, configurations, and interconnects providing different tradeoffs between capacity, speed, cost and other factors. To select

\footnotetext{
*The work was done when the author was a student at University of Rochester.

Permission to make digital or hard copies of all or part of this work for personal or classroom use is granted without fee provided that copies are not made or distributed for profit or commercial advantage and that copies bear this notice and the full citation on the first page. Copyrights for components of this work owned by others than the author(s) must be honored. Abstracting with credit is permitted. To copy otherwise, or republish, to post on servers or to redistribute to lists, requires prior specific permission and/or a fee. Request permissions from permissions@acm.org. MEMSYS, October 1-4, 2018, Old Town Alexandria, VA, USA

(C) 2018 Copyright held by the owner/author(s). Publication rights licensed to Association for Computing Machinery.

ACM ISBN 978-1-4503-6475-1/18/10 .. \$15.00

https://doi.org/10.1145/3240302.3240425
}

the right memory at the right size, it is important to measure a application's demand for memory. This demand comes from the active data usage by the application.

Traditional analysis is based on virtual memory (VM): in particular, the access bit in the page table. As a result, it's limited to page granularity. Precise analysis requires access monitoring, which is traditionally too costly for real-time use due to the frequency of memory accesses, which can be billions per second by a single thread.

Modern processors provide support to sample only the cache misses. For example, a widely used tool called perf can collect samples efficiently in near real time. On IBM Power 8 , we found that a low sample rate, e.g., a few thousand L1 misses per second, often incurred less than $1 \%$ overhead. Similar tools and hardware support can sample LLC misses and TLB misses with negligible overhead. This new hardware and tool support warrants a re-examination of fine-grained data usage analysis.

The question is: What can we re-construct from the information obtained through access sampling? Considering the problem too briefly may elicit pessimism. In $1 \%$ sampling, $99 \%$ of the trace information, selected randomly, is lost. Reconstructing, say, a photograph would be impossible after such significant loss. Another reason for pessimism is the complexity and variety of program behavior. For example, some programs may access most data only a few times, while others may access many data many times. The effect of sampling depends on such characteristics, and a low sampling ratio such as $1 \%$ may leave too little information to reconstruct the original program behavior.

In this paper, we present fine-grained data usage analysis based on the efficient access sampling available on current systems. The technique is a combination of two new models. The first is a model of the effect of sampling. It assumes the knowledge of the full trace and predicts the effect of sampling. The second model solves a real problem. Given the sampled trace, it predicts the data size of the full trace. The second model builds on the first model. We introduce a 
concept called a "motif" to enumerate possible patterns and then use the first model to evaluate and find the best choice.

We evaluate the new technique on a collection of program traces that have different characteristics in data size and reuse frequency, and different sampling ratios. We compare our technique with a previous solution. In addition, we measure the overhead in both access sampling and the data-size prediction.

A core function of memory management is memory allocation, which is done by the operating system on almost every computer today. Traditional analysis is coarse-grained. The new technique utilizes the new hardware support to provide fine-grained analysis in real time. This additional information may complement the working-set size information that an OS collects at page granularity and facilitates memory allocation based on the relative ratio of fine-grained and coarse-grained data usage (measured in real time). In addition, it can be included in an online performance tool to report the size of the actively used data at cache-line granularity. Finally, it may enable more efficient locality and cache performance analysis, whose efficiency up to now has been limited by the necessity of reuse sampling (which is discussed in Section 4).

The rest of the paper is organized as follows: Section 2 describes the new technique, and the two models it comprises. Section 3 evaluates the new technique in accuracy and efficiency and compares it with an alternative solution. Finally, Section 4 discusses related work, and Section 5 concludes.

\section{Data Usage Sampling and Prediction}

This section first defines the necessary notations and then presents the two models, the first on the sampling effect, and the second on data-size prediction.

\subsection{Notations}

A sampling ratio is the ratio between the number of samples and the length of the full trace. A trace is a sequence of accesses to data elements, which may be memory addresses or object IDs. We distinguish by calling them data accesses and data elements. We also call data elements data blocks.

A frequency histogram shows the access frequency for each data element. A frequency distribution shows how common each frequency is.

For compactness, we use a frequency-class histogram. Formally, this is a vector $\left(c_{1}, c_{2}, \ldots\right)$, where $c_{i}$ is the number of data elements that are accessed exactly $i$ times. For example, the first bucket, $c_{1}$, is the number of data elements that were accessed only once. We refer to them as unique accesses.

A frequency-class histogram is more compact than a frequency histogram. It takes only a single bucket, i.e., the first bucket $c_{1}$, in the frequency-class histogram to count all unique accesses but one bucket per unique access in the frequency histogram. If we discard the identity of data elements, as we do in this paper, the two types of histograms can be converted to each other. They are equivalent.

We write the histograms (using Latin letters) as follows: $m_{i}$ is the full-trace frequency histogram, $c_{i}$ is the full-trace frequency-class histogram. The two corresponding histograms for the sampled trace use Greek letters: $\mu_{i}$ and $\gamma_{i}$.

If the histogram is predicted, this is indicated with a prime. For example, $\gamma_{i}^{\prime}$ is the predicted frequency-class histogram of the sampled trace. Finally, we may use a distribution, which is the same as a histogram except that each bucket is normalized, i.e., as a portion of the trace length in a frequency distribution and as a portion of the total data size in a frequency-class distribution.

All histograms may be converted into a distribution. A frequency distribution is represented by $P(\bullet)$. For example for the full trace, $c_{i}$ is the frequency-class histogram, and $P\left(c_{i}\right)$ the frequency-class distribution. Each $P\left(c_{i}\right)$ shows the portion of data blocks that are accessed $i$ times in the full trace. The following table shows the symbols used in the rest of the paper.

\begin{tabular}{|c|c|}
\hline Symbol & Definition \\
\hline$p$ & the sampling ratio \\
\hline$n, n_{s}$ & $\begin{array}{l}\text { the length of the full and sampled trace, } \\
n_{s}=n p\end{array}$ \\
\hline$d, d_{s}$ & $\begin{array}{l}\text { the data size of the full and sampled } \\
\text { trace }\end{array}$ \\
\hline$m_{i}, \mu_{i}$ & $\begin{array}{l}\text { frequency histogram: block } i \text { is ac- } \\
\text { cessed } m_{i} \text { times in the full and } \mu_{i} \text { times } \\
\text { in the sampled trace }\end{array}$ \\
\hline$m_{\max }$ & $\begin{array}{l}\text { the highest access frequency of a single } \\
\text { block in the full trace }\end{array}$ \\
\hline$c_{i}, \gamma_{i}$ & frequency-class histogram: $c_{i}$ blocks \\
\hline & $\begin{array}{l}\text { are accessed } i \text { times in the full trace, } \\
\text { and } \gamma_{i} \text { blocks are in the sampled trace }\end{array}$ \\
\hline$P(\bullet)$ & a distribution: $P\left(c_{i}\right)=\frac{c_{i}}{d}, P\left(\gamma_{i}\right)=\frac{\gamma_{i}}{d_{s}}$ \\
\hline
\end{tabular}

A related problem is sampling in a multiset. A multiset may contain multiple instances of an element. The number of its instances is the multiplicity of the element. For example, $\{a, a, b\}$ is a multiset, and the multiplicity of $a$ is 2 . For the full multiset, the frequency histogram $m_{i}$ shows the multiplicity $m_{i}$ for each element $i$. The frequency-class histogram shows the count of elements $c_{i}$ with the same multiplicity $i$. For the sampled multiset, the two histograms are $\mu_{i}, \gamma_{i}$ respectively. As explained earlier, $m_{i}$ and $c_{i}$ are mutually convertible, as are $\mu_{i}$ and $\gamma_{i}$.

\subsection{Predicting The Effect of Sampling}

The goal of the prediction can be regarded as the following: given an original trace and the sampling ratio $p$, predict the frequency histogram of the sampled trace. 
It is intuitive to picture a bin with colored balls. A full trace with $n$ accesses to $d$ data blocks can be represented as a bin with $n$ balls of $d$ different colors. We sample a set of balls with the sampling ratio. For example, assume a bin with ten balls, where different numbers denote different colors: 123 3214565 . After 50\% sampling, the extracted numbers may be: 12345 . There are 5 colors and one ball for each color. The problem is whether we can predict the number of colors and the number of balls of each color in the sample from the content of the bin and the sampling ratio.

Consider a simplified example distribution, where $50 \%$ of balls all have the same color, and the other $50 \%$ are each colored differently. If we sample $1 \%$ of the balls, half of the sampled balls have one color, and the remaining half all have different colors. The total number of colors is therefore half the number of sampled balls plus one.

Here we consider the general problem. The full trace is sampled with probability $p$, which yields $n_{s}=n p$ samples. Algorithm 1 shows the prediction of the sampling effect. It takes the frequency-class histogram of the full trace $c_{i}$, the sampling ratio $p$ and gives the predicted frequency-class histogram of the sampled trace $\gamma_{i}^{\prime}$.

Input: sampling ratio $p$

Input: the frequency-class histogram of the full trace $c_{i}$

Output: the predicted frequency-class histogram of the sampled trace $\gamma_{i}^{\prime}$, initially $\gamma_{i}^{\prime}=0$ for $i=1, \ldots, m_{\max }$

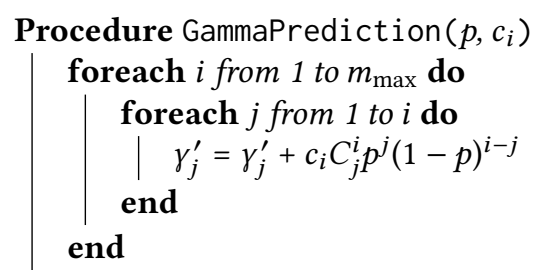

Algorithm 1: The algorithm to predict the frequency-class histogram of the sampled trace using the frequency-class histogram of the full trace

Algorithm 1 has two nested loops. The first loop iterates through all the buckets of $c_{i}$ for $i=1, \ldots, m_{\max }$. For each $c_{i}$, the second loop computes the distribution of these $c_{i}$ data blocks into the $\gamma_{j}^{\prime}$ buckets for $j=1, \ldots, i$. It is obvious that $j$ is at most $i$, since a block accessed $i$ times in the full trace cannot show up more than $i$ times in the sampled trace.

As the result of sampling, a portion of $c_{i}$ becomes part of $\gamma_{j}$. This portion is predicted by $C_{j}^{i} p^{j}(1-p)^{i-j}$. The formula is based on randomness. There are $C_{j}^{i}$ ways to choose which $j$ of the $i$ accesses to sample, and the probability of sampling only $j$ accesses is $p^{j}(1-p)^{i-j}$, that is, exactly $j$ accesses are sampled, and $i-j$ accesses are not.
We make three observations. First, the formula can be implemented very efficiently. The calculation $C_{j}^{i} p^{j}(1-p)^{i-j}$ does not depend on the input, can be done before hand, and becomes a constant in actual implementation. Second, the input can be either $c_{i}$, as the algorithm shows, but it can also be $P\left(c_{i}\right)$, in which case the algorithm returns $P\left(\gamma_{j}^{\prime}\right)$ instead of $\gamma_{j}^{\prime}$. This is in fact how we will use the algorithm in the second model (to be discussed later with Algorithm 2). Third, the nested loop is a linear transformation based on constant co-efficients. We will discuss the linear inverse problem in Section 3.5.

\subsection{Predicting Full-trace Data Size}

Given the sampled trace and the sampling ratio, this section presents a method that predicts the data size of the full trace. The basic problem is to recover the information lost due to sampling.

We model the effect of sampling using the prediction technique just presented in Section 2.2. If we had the correct frequency histogram of the full trace, we would be able to predict the frequency histogram of the sampled trace. In other words, we can now check the correctness of the frequency histogram of the full trace.

The ability to check for correctness solves half of the problem. We still need to "guess" the frequency histogram so that we can check for correctness. The prediction technique systematically enumerates possible histograms, selects the most correct histogram, and computes the data size.

It is too costly to enumerate all possible frequency histograms. We restrict the search space as follows. The first technique is normalization - we use the frequency distribution instead of the frequency histogram. The second is restriction. We consider a specific pattern, which we call a motif, that restricts the space of distributions to a group of possible "shapes". The third is parameterization. We use three parameters to define a specific frequency distribution.

The motif is a pattern of the frequency-class distribution, shown in Figure 1. It divides the buckets into two groups: the first group of buckets follow an exponential distribution and the second part is constant. The motif has three parameters: the total number of buckets $n$, the break point $b p$ (i.e. the number of buckets in the first group), and the height ratio $h$. The height ratio is the ratio of the first bucket to the last bucket in the first group. All buckets of the second group has the same height as the last bucket of the first group. Let the motif distribution be $P\left(c_{i}^{\prime \prime}\right)$. The motif distribution has first $P\left(c_{1}^{\prime \prime}\right)=h$ and then at the end $P\left(c_{d p}^{\prime \prime}\right), \ldots, P\left(m_{\max }\right)=1 / h$.

Algorithm 2 shows the algorithm we use. It has three nested loops to enumerate the parameter space of the motif. For each parameter combination, it calls BuildMotif to construct a full-trace frequency distribution $P\left(c_{i}^{\prime \prime}\right)$. Then it uses Algorithm 1 (by calling GammaPrediction) to use the sampling model and predict the sampled-trace frequency 


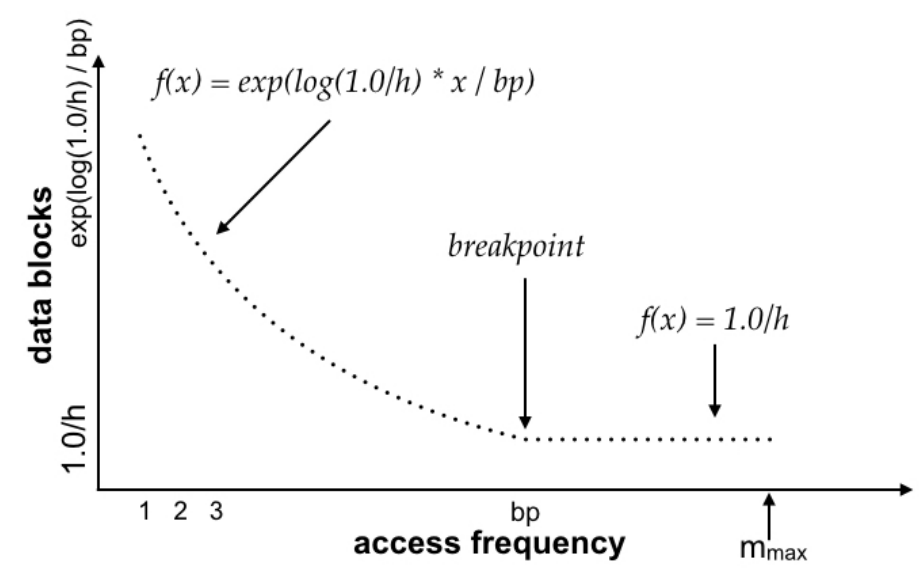

Figure 1. Illustration of the model motif with three parameters: $h, b p, m_{\max }$

distribution $P\left(\gamma_{i}^{\prime \prime}\right)$. This is compared with the observation $P\left(\gamma_{i}\right)$. The error $l$ is computed by taking the total (absolute) difference of the first 5 buckets. The reason for choosing the first 5 is discussed in later with Figure 3. Through the numeration of the parameter space, Algorithm 2 finds the best $P\left(c_{i}^{\prime}\right)$ with the lowest error $\min _{l} \cdot{ }^{1}$ In the last step, it computes the data size and returns it as the result of the prediction.

\section{Evaluation}

This section first describes the experimental setup, evaluates the two models of the new technique, discusses two alternative solutions, and finally measures the efficiency of access sampling and data-size prediction.

\subsection{Methodology and Benchmarks}

The traces were collected using an IBM server, model S822L, equipped with POWER8 processors. Each POWER8 core has $64 \mathrm{~KB}$ L1 data cache, $512 \mathrm{~KB}$ L2 cache, and $8 \mathrm{M}$ share of L3 cache. The OS is Linux 3.19.0. The traces were collected using the perf tool, which sets the sampling parameters through the processor interface to collect a sequence of samples from L1 cache misses and dumps the collected samples periodically. The operating system was modified to expose the required processor interface for sampling L1 misses.

We were studying parallel program performance and chose the NAS benchmark suite, which is representative of scientific computing. NAS has 10 benchmarks. In the following evaluation, we include all except for two, EP and IS. EP stands for "embarrassingly parallel". Its data size is too small to be interesting. IS has a large data size but the access frequency for almost all data is $1 .^{2}$

\footnotetext{
${ }^{1}$ The minimal error $\min _{l}$ is initialized to 2 , which is the largest possible difference between two distributions.

${ }^{2}$ Predicting IS data size is simply $d^{\prime}=d_{s} / p$.
}

Trace Sampling The traces were collected using perf as described in Section 3.6. For each program, we used the longest trace as the full trace and randomly sample the full trace to generate the sampled trace.

Model Implementation Our model motif has three parameters. We use Algorithm 2 to tune them for each program. In our implementation, we search $m_{\max }^{\prime}$ from 50 to 250 (inclusive) at the step size 50 and iterate $h$ for the following 6 values: $100,200,500,1000,2000,5000$. The accuracy of the motif is evaluated using the first 5 buckets, since they include most data blocks in a sampled trace (as we will discuss with Figure 3). We found these implementation parameters sufficient for our test programs and believe that they can be changed fairly easily if needed for different types of workloads, e.g. access traces rather than L1 miss traces.

\subsection{Sampling Effect Prediction}

The prediction result of the sampling effect is shown in Figure 3. Each graph shows one program. It shows the frequencyclass histogram in 6 buckets. The first 5 show $P\left(\gamma_{1}\right), \ldots, P\left(\gamma_{5}\right)$, and the last bucket shows the sum of the rest, i.e. $\sum_{i=6} P\left(\gamma_{i}\right)$. The predicted histogram is shown in the same way in 6 buckets.

The model of sampling effect in Section 2.2 is extremely accurate. The prediction based on the full trace information closely matches the histogram of the sampled trace in all 6 buckets in all 8 programs. We have also calculated the accuracy as the ratio of the prediction to the actual for the first 5 buckets. Both the prediction and the actual are calculated by the sum. Precisely, the accuracy is as follows:

$$
\frac{\text { predicted }}{\text { measured }}=\frac{\sum_{i=1}^{5} P\left(\gamma_{i}^{\prime}\right)}{\sum_{i=1}^{5} P\left(\gamma_{i}\right)}
$$


Fine-grained Data Usage Analysis by Access Sampling
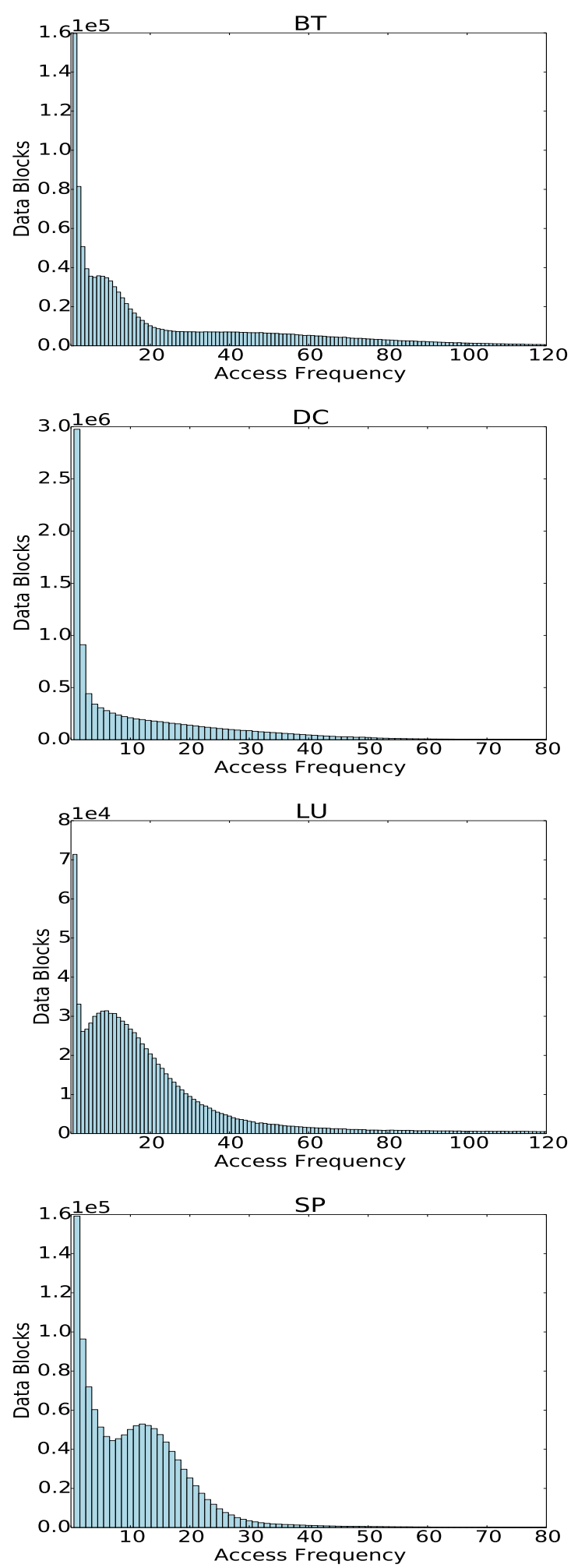

MEMSYS, October 1-4, 2018, Old Town Alexandria, VA, USA
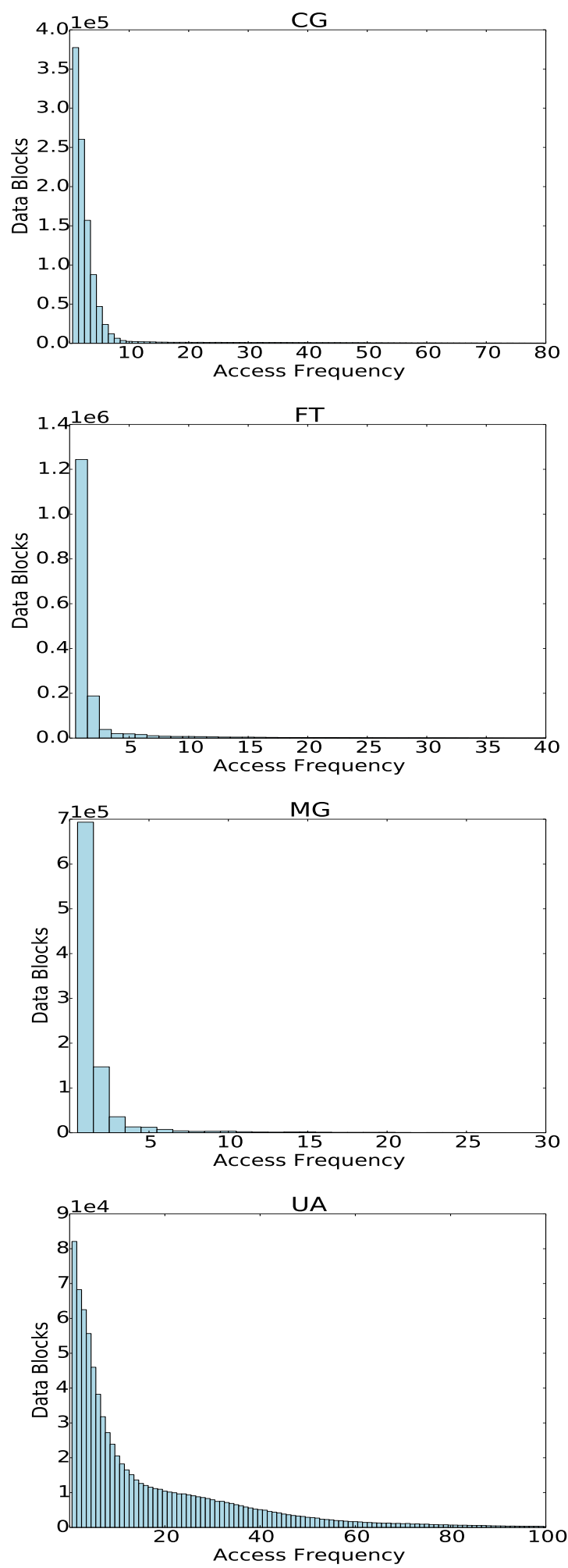

Figure 2. The frequency-class histogram of the full trace $c_{i}$. 

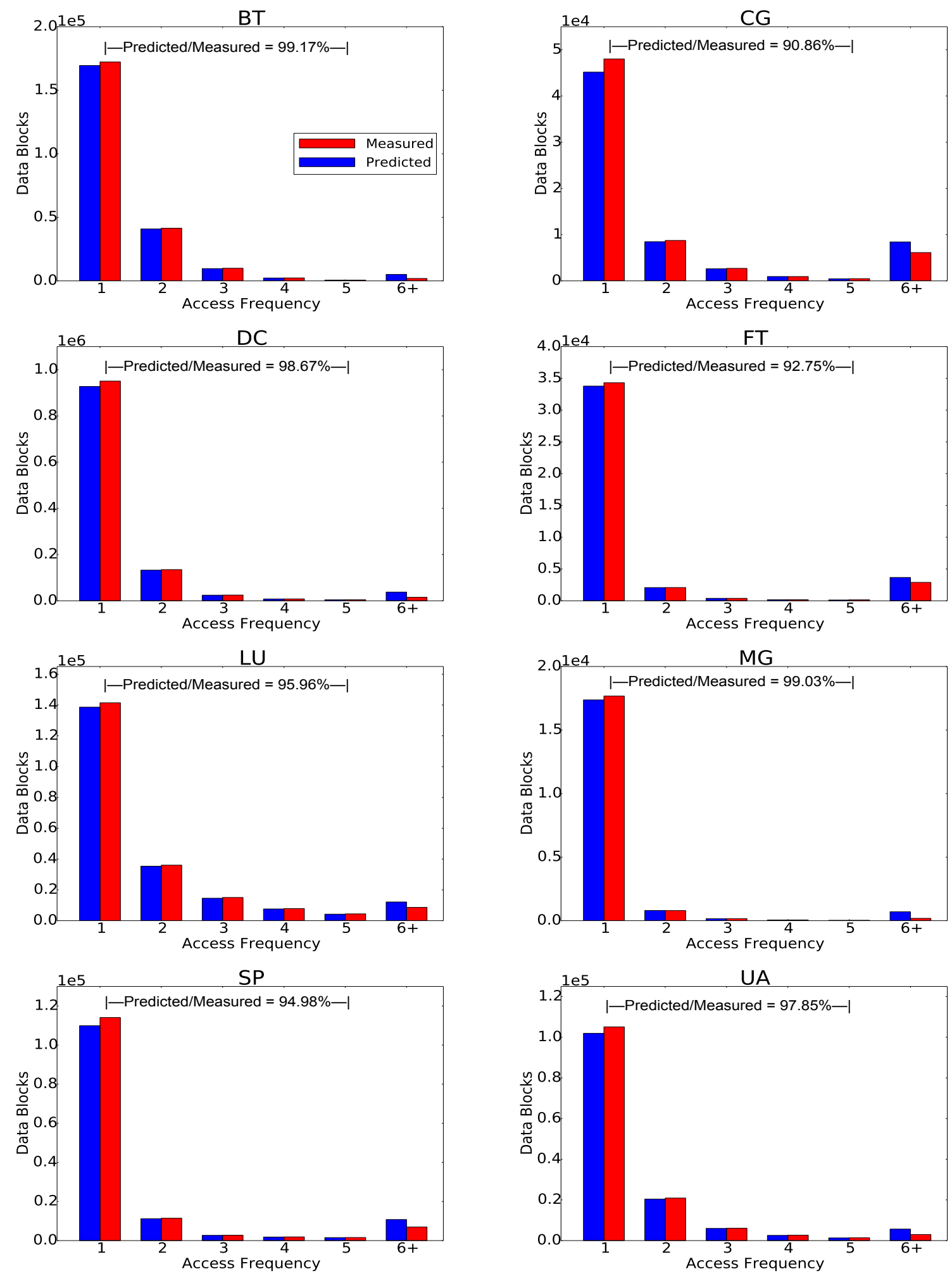

Figure 3. Predicted and measured frequency-class histograms of the sampled trace: $\gamma_{i}, \gamma_{i}^{\prime}$. Each graph shows the prediction accuracy for the first five buckets, which ranges from $91 \%$ to $99 \%$. 
Input: sampling ratio $p$

Input: sampled frequency-class histogram $\gamma_{i}$

Output: prediction of data size of the full trace $d^{\prime}$
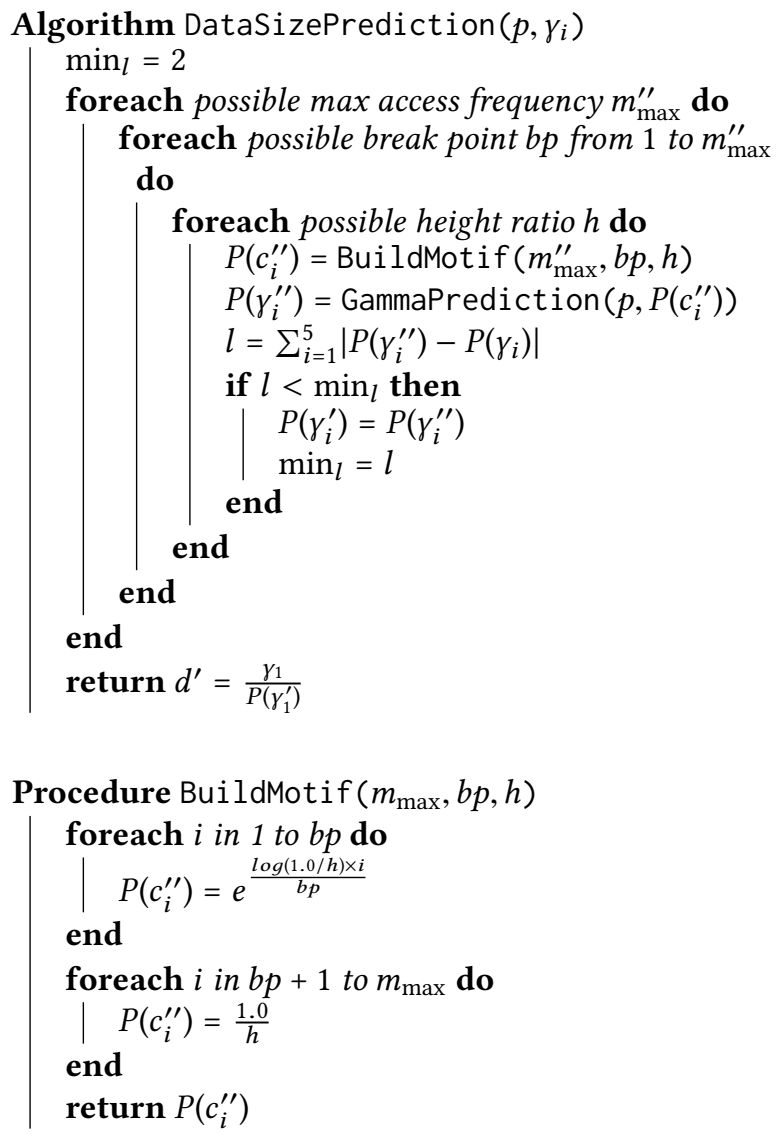

Algorithm 2: The algorithm to predict the full-trace data size using the frequency-class histogram from the sampled trace.

Each graph in the figure shows the accuracy of prediction for the first 5 histogram buckets, which ranges from $91 \%$ in CG to $99 \%$ in BT. On average, the prediction accuracy is $96 \%$.

Furthermore, Figure 3 shows that most data blocks in the sampled traces are accessed 5 times or fewer, which is the reason that Algorithm 2 evaluates each motif candidate using (its prediction of) the first 5 buckets.

For a more complete view on the effect of sampling, we show the frequency-class histogram of the full trace in Figure 2. In both figures, the first bucket in a frequency-class histogram shows the number of unique accesses. The bucket $c_{1}$ counts the unique accesses in the full trace, and $\gamma_{1}$ the unique accesses in the sampled trace. The highest counts for the full trace are MG 73\%, FT 76\%, and CG 35\%. The lowest are LU $7 \%$ and UA $10 \%$. In sampled traces, the highest are MG $93 \%$, FT $86 \%$. However, the relative order of CG and UA are reversed (in the sampled trace) with CG 72\%, lower than UA 76\%. The lowest is LU 66\%. The sampling model of Section 2.2 predicts these effect accurately.

\subsection{Data Size Prediction}

Table 1 shows the accuracy of data-size prediction described in Section 3.3. It shows the results separately for $1 \%$ and $10 \%$ sampling in two tables. Each table shows the prediction error, the error of the motif $\min _{l}$, and the three parameters of the motif: the breakpoint $b p$, the total number of buckets $m_{\max }$, and the height ratio $h$.

Using $1 \%$ sampling, the best result is DC, whose error is less than $4 \%$, and the worst result comes from LU, whose error is more than $200 \%$. Across 8 programs, the arithmetic mean is $69 \%$ and the geometric mean is $33 \%$. Using $10 \%$ sampling, the best result is SP, which is almost $100 \%$ accurate, and the worst result is FT, whose error is $23 \%$. The arithmetic mean of the prediction error is $11 \%$ and the geometric mean only $6 \%$.

The prediction is much more accurate using $10 \%$ sampling than using $1 \%$ sampling, showing the importance of having 10 times more samples and, equally importantly, the fact that our technique makes use of the additional information to make 5 times more accurate predictions (33\% vs 6\%).

We observe two reasons for errors, shown by the tests. One pair of tests, LU and SP, have large errors with $1 \%$ sampling. We found that the smallest $\min _{l}$ is not necessarily the best motif in that case. Their errors, however, disappear when more samples are used. Another pair of tests, MG and FT, have the lowest average data reuse among the 8 benchmarks. They have the largest under-prediction with $1 \%$ sampling, and the errors are reduced but not eliminated with $10 \%$ sampling. Their low average data reuse suggests poor locality and possibly the weakness in modeling such programs by our technique.

Table 1 also shows that with more samples, the motif selection uses more of the parameter space. For example, the number of buckets in the motif $m_{\max }$ is almost all 250 in the first table, but it covers the full range of tuning from 50 to 250 in the second table.

\subsection{Comparison with Multifractal and Good-Turing}

We compare our new motif based technique with two previous solutions. We first describe the earlier solutions and then evaluate their prediction accuracies.

Good-Turing Estimation Good-Turing frequency estimation is a technique widely used in statistics and Natural Language Processing (NLP). Given the past observed sequences of objects, the Good-Turing estimation can give the probability of the next objects that are not yet seen. A version of Good-Turing is applied to our prediction problem as follows:

$$
d=\frac{\gamma_{1}}{n_{s}}\left(n-n_{s}\right)+d_{s}
$$


Table 1. The benchmarks, motif errors and parameters for data-size prediction from the $1 \%$ sampling (left table) and $10 \%$ sampling (right table)

\begin{tabular}{|c|r|r|r|r|r|}
\hline Prog. & Error & $\min _{l}$ & $b p$ & $m_{\max }$ & $h$ \\
\hline BT & $-20.02 \%$ & 0.00453545 & 140 & 200 & 100 \\
\hline CG & $37.407 \%$ & 0.0831957 & 16 & 250 & 5000 \\
\hline DC & $-3.64 \%$ & 0.0133038 & 61 & 200 & 500 \\
\hline FT & $-49.83 \%$ & 0.0693782 & 30 & 250 & 5000 \\
\hline LU & $232.66 \%$ & 0.0427919 & 18 & 250 & 2000 \\
\hline MG & $-56.84 \%$ & 0.0133886 & 33 & 150 & 5000 \\
\hline SP & $145.19 \%$ & 0.0542505 & 24 & 250 & 5000 \\
\hline UA & $-5.75 \%$ & 0.0214397 & 82 & 250 & 200 \\
\hline arith. & $68.92 \%$ & \multicolumn{4}{|l}{} \\
\cline { 1 - 2 } geo. & $33.27 \%$ &
\end{tabular}

\begin{tabular}{|c|r|r|r|r|r|}
\hline Prog. & Error & $\min _{l}$ & $b p$ & $m_{\max }$ & \multicolumn{1}{|c|}{$h$} \\
\hline BT & $-6.61 \%$ & 0.104925 & 114 & 250 & 100 \\
\hline CG & $-8.40 \%$ & 0.0275941 & 15 & 100 & 500 \\
\hline DC & $-23.29 \%$ & 0.0311854 & 67 & 100 & 100 \\
\hline FT & $-23.29 \%$ & 0.0211737 & 11 & 50 & 1000 \\
\hline LU & $4.02 \%$ & 0.0674798 & 106 & 200 & 100 \\
\hline MG & $-19.18 \%$ & 0.0230952 & 11 & 50 & 2000 \\
\hline SP & $0.18 \%$ & 0.0357039 & 84 & 100 & 200 \\
\hline UA & $-4.44 \%$ & 0.055972 & 80 & 200 & 100 \\
\hline arith. & $11.18 \%$ & \multicolumn{5}{|l}{} \\
\hline geo. & $6.08 \%$ & &
\end{tabular}

Table 2. Prediction by Multifractal and Good-Turing at $1 \%$ and $10 \%$ sampling ratios

\begin{tabular}{|c|c|c|c|c|c|c|c|c|}
\hline \multirow[b]{3}{*}{ Prog. } & \multicolumn{6}{|c|}{ Multifractal prediction } & \multicolumn{2}{|c|}{ Good-Turing prediction } \\
\hline & \multicolumn{3}{|c|}{$1 \%$ sampling } & \multicolumn{3}{|c|}{$10 \%$ sampling } & $1 \%$ sampling & $10 \%$ sampling \\
\hline & Error & $p$ & $k$ & Error & $p$ & $k$ & Error & Errol \\
\hline BT & $195 \%$ & 0.835579 & 26 & $141 \%$ & 0.829582 & 25 & $1380 \%$ & $137 \%$ \\
\hline CG & $6 \%$ & 0.895368 & 28 & $6 \%$ & 0.895375 & 28 & $347 \%$ & $88 \%$ \\
\hline $\mathrm{DC}$ & $406 \%$ & 0.985844 & 172 & $174 \%$ & 0.973967 & 93 & $800 \%$ & $125 \%$ \\
\hline FT & $-66 \%$ & 0.847066 & 23 & $-20 \%$ & 0.875921 & 29 & $111 \%$ & $35 \%$ \\
\hline LU & $370 \%$ & 0.835025 & 28 & $183 \%$ & 0.816964 & 25 & $1359 \%$ & $173 \%$ \\
\hline MG & $8 \%$ & 0.940147 & 58 & $13 \%$ & 0.94154 & 60 & $86 \%$ & $36 \%$ \\
\hline SP & $262 \%$ & 0.900235 & 38 & $181 \%$ & 0.889237 & 34 & $1125 \%$ & $161 \%$ \\
\hline UA & $365 \%$ & 0.884832 & 34 & $162 \%$ & 0.861692 & 28 & $811 \%$ & $70 \%$ \\
\hline arith. & $210 \%$ & & & $110 \%$ & & & $752 \%$ & $86 \%$ \\
\hline geo. & $99 \%$ & & & $62 \%$ & & & $509 \%$ & $89 \%$ \\
\hline
\end{tabular}
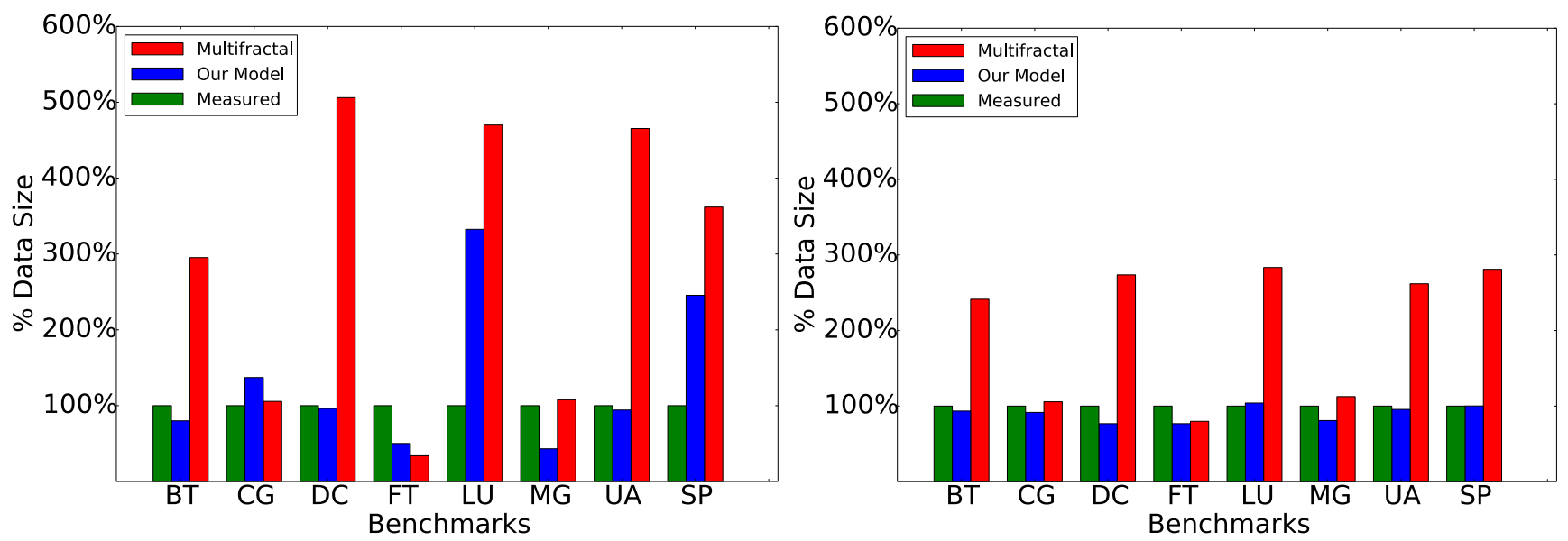

Figure 4. Comparison between Multifractal and our technique at sampling ratio $1 \%$ (left) and 10\% (right). Good-Turing is not shown, but its errors are reported in Table 2. 
The ratio $\frac{\gamma_{1}}{n_{\mathrm{s}}}$ is the probability of seeing a new object in the next access. Good-Turing calculates the expected number of new objects among unseen accesses and adds the the number of objects in the sampled trace $d_{s}$. Note that in this model, any sampled object may be contained in the predicted set.

Multifractal A multifractal distribution has two parameters, the bias $p$ and order $k$. When the order is 1 , the distribution has two buckets with probability $p$ and $1-p$. At each successive order, each bucket is split into two with the same divide of $p$ and $1-p$. Schroeder [9] showed many uses of multifractals in modeling the "80-20 law" such as photon distributions in physics, commodities distributions on earth, and salary distributions.

Faloutsos, Matias and Silberschatz developed a technique called the multifractal method [1996]. It uses an algorithm to estimate the bias $p$ and order $k$ based on three inputs of a sampled multiset: the number of records, the largest multiplicity, and the data size. These correspond to $n_{s}, m_{\max }, d_{s}$ using the symbols in this paper. Once $p, k$ are determined, the data size of the full trace is computed by Eq. (1).

$$
d^{\prime}=\sum_{a=0}^{k} C_{a}^{k}\left(1-\left(1-p^{k-a}(1-p)^{a}\right)^{n}\right)
$$

Since the largest probability is $p^{k}$, the initial bias is estimated by $p=\left(m_{\max } / n\right)^{1 / k}$. Then, the tuning process uses Eq. (1), by using $n_{s}$ instead of $n$, to predict the data size in the sampled trace $d_{s}^{\prime}$, and compares the prediction with the actual $d_{s}$. The equation starts as an underestimate and increases monotonically with $k$. The original method of Faloutsos et al. was to increase $k$ until the predicted data size $d_{s}^{\prime}$ matches with observation $d_{s}$ "within a desired tolerance $\epsilon$ ". In our implementation, we chose $k$ that gave the most accurate prediction, i.e. minimal difference between $d_{s}^{\prime}$ and $d_{s}$.

Comparisons Table 2 shows the prediction error and the model parameters of Multifractal first and Good-Turing next.

For Multifractal, the skew factor $p$ is at least 0.82 and near 0.9 in most cases, showing that our test traces have highly skewed distributions. Multifractal over-predicts in most cases. The best result is CG, whose error is around $6 \%$ in both $1 \%$ and $10 \%$ sampling. The worst is DC and LU respectively in $1 \%$ and $10 \%$ prediction, whose errors are more than $400 \%$ and $183 \%$. The average errors, for both arithmetic and geometric means and in both $1 \%$ and $10 \%$ sampling, are much greater than the errors of our technique.

The difference is shown most clearly when the predictions by the two methods are plotted side by side in Figure 4 . The figure has two graphs for two sampling ratios. The predictions by our technique are much closer to the actual, and the accuracy of our technique improves by a much greater degree when the sampling ratio is increased from $1 \%$ to $10 \%$.

Good-Turing does not work well, especially at $1 \%$ sampling. Part of the reason is that we not only care about whether the next object has occurred before, but we are also concerned by the "distinctness" of the next object, which can lead to double counting the same object in the size prediction. Still, we can observe from the table that with more data, the model becomes more accurate. In fact, the average arithmetic error, $86 \%$, is lower than Multifractal, $110 \%$. However, the average error of our model, $11 \%$, is significantly lower than both of the previous methods.

\subsection{Solution by Linear Inversion}

Algorithm 1 computes $\gamma_{i}$ from $c_{i}$ using a linear conversion. Let $M[i, j]=C_{j}^{i} p^{j}(1-p)^{i-j}$ for $j \leq i$ and $M[i, j]=0$ otherwise. Effectively, $\gamma=M c$. It is tempting to consider solving the linear inverse problem to use the sampled trace and predict the full trace, that is, $c=M^{-1} \gamma$.

This solution does not work. We can show it as follows. Take $c_{i}$ and compute $\gamma_{i}$. Then we take this $\gamma_{i}$ and solve the inverse equation. When the number of buckets $m_{\max }$ is up to 20 , the inversion works. We obtain the original $c_{i}$. However, when $m_{\max }=50$, the inversion process produces numerical overflow, even though we use double precision floating-point numbers.

The reason is the type of co-efficients in $M[i, j]$. Consider $j=50$ and $p=0.01$. Then $p^{j}=1 e^{-100}$. Such small co-efficients are fine when used in multiplication in Algorithm 1. However, they will be used as denominators in the inverse solution and cause numerical overflow. The problem is actually worse. If the linear inversion was used, the input is noisy, and any small deviation can be amplified astronomically by these small co-efficients. Even if we use infinite-precision numbers, the inversion model can easily predict absurd data sizes such as $10^{50}$.

\subsection{Sampling and Modeling Costs}

Sampling is performed using a modified version of the perf tool implemented in the Linux kernel. It is configured to sample at a frequency specified either as a rate, i.e. the number of samples per second, or periodically, i.e. taking a sample at every $k$ th event. The hardware events sampled are PM_LD_MISS_L1 and PM_ST_MISS_L1. The first counts the load misses and the second the store misses, both of the L1 data cache.

Table 4 shows the overhead of sampling for different sampling ratios. The overhead is measured by the increase in wall-clock time of the full execution compared to the execution without any sampling.

In fact, $1 / 1$ sampling is to collect all L1 misses, not actually "full trace". In comparison, collecting the full access trace requires instrumentation and would increase the running time by an order of magnitude. In addition, the time in Table 4 is reported for the perf tool, which is a "best effort" tool. When the overhead exceeds a threshold, it drops samples. 
Table 3. Time overhead of data-size prediction running on MacBook Pro Early 2015 (2.7 GHz Intel Core i5 processor and $8 \mathrm{~GB} 1867 \mathrm{MHz}$ DDR3 memory)

\begin{tabular}{|c|c|c|}
\hline Benchmark & $\begin{array}{c}\text { 1\% sampling } \\
\text { pred. time(s) }\end{array}$ & $\begin{array}{c}\text { 10\%sampling } \\
\text { pred. time(s) }\end{array}$ \\
\hline BT & 3.00 & 2.98 \\
\hline CG & 3.22 & 3.17 \\
\hline DC & 9.01 & 9.95 \\
\hline FT & 2.87 & 2.90 \\
\hline LU & 2.89 & 2.99 \\
\hline MG & 2.88 & 2.83 \\
\hline SP & 3.01 & 2.93 \\
\hline UA & 2.91 & 2.93 \\
\hline
\end{tabular}

Table 4. The time overhead of L1 miss sampling on IBM POWER8

\begin{tabular}{|c|l|l|l|l|l|l|l|}
\hline \multirow{2}{*}{$\begin{array}{c}\text { Pro- } \\
\text { grams }\end{array}$} & \multicolumn{7}{|c|}{ Ratio of full exe. time } \\
\cline { 2 - 8 } & $1 / 1$ & $1 / 10$ & $1 / 100$ & $1 / 1 \mathrm{~K}$ & $1 / 5 \mathrm{~K}$ & $1 / 10 \mathrm{~K}$ & $1 / 15 \mathrm{~K}$ \\
\hline BT & 1.190 & 1.173 & 1.140 & 1.093 & 1.032 & 1.012 & 1.004 \\
\hline CG & 1.168 & 1.193 & 1.176 & 1.151 & 1.151 & 1.108 & 1.009 \\
\hline DC & 1.347 & 1.215 & 1.221 & 1.072 & 0.959 & 1.066 & 1.381 \\
\hline FT & 1.204 & 1.200 & 1.178 & 1.110 & 1.095 & 1.104 & 1.080 \\
\hline LU & 1.200 & 1.212 & 1.190 & 1.115 & 1.065 & 1.039 & 1.029 \\
\hline MG & 1.205 & 1.204 & 1.125 & 1.098 & 1.051 & 1.050 & 1.053 \\
\hline SP & 1.190 & 1.170 & 1.118 & 1.073 & 1.038 & 1.027 & 1.016 \\
\hline UA & 1.208 & 1.172 & 1.091 & 1.048 & 1.025 & 1.018 & 1.015 \\
\hline
\end{tabular}

The actual samples collected are often far lower than $100 \%$. In fact, similar overheads mean a similar number of samples collected, and the actual samples collected in 1/1 sampling runs are much closer to $1 \%$ than to $100 \%$.

Reading vertically, the overhead of the same sampling ratio can vary significantly from one program to another, as programs have a different miss rate, i.e. number of L1 misses per second. It is interesting that the practical result can be contrary to the theory. For example, the overheads of CG and LU at 1 is smaller than those in 10. Theoretically, the higher the sampling frequency is, the heavier the overhead should be.

Modeling Overhead Table 3 shows the time overhead of the data-size prediction, which we can see is very light. The results were collected on a MacBook Pro, and most of the running time was around 3 seconds. One outlier is DC, which takes more than 9 seconds. Part of the reason is that it has a significant larger histogram and a longer trace than the other seven benchmarks. Also, we can notice that with more data, there isn't significant increase in the overhead by comparing the analysis times of $1 \%$ and $10 \%$ sampling. Combining the information of Table 4 and Table 3, it is safe to draw the conclusion that the technique of access sampling and the data-size prediction described in the paper has a low overhead.

\section{Related Work}

The technique in this paper analyzes the effect of access sampling, which is related to two previous areas of work on multiset and reuse sampling.

Multiset Sampling In database queries, it is often useful to estimate a frequency histogram, for example, to estimate the number of products that account for $50 \%$ of the sales. Ioannidis and Poosala [7] used a high biased histogram, which records the frequencies of a few most frequent items and makes the uniformity assumption for the rest. Faloutsos et al. [5] observed that high biased histograms "seem to be the state of the art in current commercial systems," and studied an improvement based on multifractals, as described and evaluated in Section 3.4. A multifractal distribution is parameterized by the bias $p$ and the level of fractal $k$. Faloutsos et al. [5] gave a method to estimate the best $p, k$. By assuming the same distribution for the whole set and the sampled set, the multifractal is then used to estimate the features of the whole set using the observation of a sub-set.

Multifractal was designed to solve the general problem of multiset sampling, of which access sampling is a special case. As shown in Section 3.4, multifractal can be used in access sampling and makes accurate prediction in two of the test programs. In comparison, the new motif based technique is designed especially for access sampling and is more accurate. At $10 \%$ sampling ratio, the geometric mean error is $6 \%$ for the new technique but $62 \%$ for multifractal. Hence, the evaluation shows that the motif based technique is superior for access sampling.

Multifractal does not consider the effect of sampling. It assumes that the full set and the sampled set have the same skewed distribution. The motif-based technique, however, builds on a model to predict the sampling effect (Section 2.2), which has been shown to be over $96 \%$ accurate on average (Section 3.2). Multifractal, however, can be used to estimate not only the data size but also other frequency moments [5]. Other frequency moments remain the future work for the new technique.

Reuse Sampling Reuse sampling is widely used in locality and cache performance analysis. In profiling analysis, Beyls and D'Hollander [1] used hold-and-sample, which selects an data item and then samples its reuses. Eklov and Hagersten [4] developed StatStack, which samples consecutive pairs of accesses to the same data. The same method was used by $\mathrm{Hu}$ et al. [6] to measure the average eviction time (AET). AET has recently been used in storage and memory caching $[2,14]$ and in compiler locality analysis [3]. Tam et al. [10] developed a tool called RapidMRC, which uses the hardware support on the IBM Power processor to find 
the reuse of a sampled data block. A set of techniques used bursty sampling for locality analysis by taking a fraction of a trace periodically and measuring the footprint $[12,13]$. In cache design, Qureshi and Patt [8] developed utility-based cache partitioning and an utility monitor (UMON) which samples the accesses at selected cache sets. In file-system design, Waldspurger et al. [11] developed spatial sampling, which extracts the accesses to a set of file blocks and simulates the sample accesses to obtain cache performance for the whole trace.

All these techniques sample reuses. Reuse sampling must monitor every access to the same data. Access sampling differs in that it may see only a subset of accesses and not intervening ones to the same data. Access sampling is easier and more efficient to implement both in software and hardware and therefore has a lower overhead than reuse sampling. Access sampling is also more flexible and can take samples from any level of a memory hierarchy. For example, taking L2 misses makes no difference to access sampling compared to taking L1 misses except for the difference in sampling ratio. Reuse sampling, however, can be used to compute the cache performance, which access sampling cannot do yet.

\section{Summary}

This paper has presented a new technique to model the effect of access sampling. It includes first an accurate model to predict the effect of sampling, that is, predicting the frequency histogram of the sampled trace using the frequency histogram of the full trace. Using the first model, it uses a motif-based technique to predict the data size of the full trace using the frequency histogram of the sampled trace. The evaluation shows that the model of sampling is highly accurate, over $96 \%$ on average. The accuracy of data-size prediction depends on the sampling ratio. The average error is $33 \%$ at $1 \%$ sampling and $6 \%$ at $10 \%$ sampling. The new technique is 3 times and 5 times more accurate than a previous technique based on multifractals, whose average errors are $99 \%$ and $62 \%$ for $1 \%$ and $10 \%$ sampling respectively. The predictions compared with Good-Turing Estimation are 11 and 8 times more accurate respectively.

\section{Acknowledgments}

The authors would like to thank Jacob Brock for early participation, Daniel Gildea for help with the Good-Turing Test, Daniel Stefankovic for help with statistical methods, Yufei Ding for supporting the travel, Wesley Smith for proof reading, Bruce Jacob for shepherding the paper, and the comments and suggestions of the anonymous reviewers. The first author was supported by funding from IBM Center for
Advanced Studies. Other support includes the National Science Foundation (Contract No. CCF-1717877, CCF-1629376).

\section{References}

[1] Kristof Beyls and Erik H. D’Hollander. 2006. Discovery of localityimproving refactoring by reuse path analysis. In Proceedings of High Performance Computing and Communications. Springer. Lecture Notes in Computer Science, Vol. 4208. 220-229.

[2] Daniel Byrne, Nilufer Onder, and Zhenlin Wang. 2018. mPart: missratio curve guided partitioning in key-value stores. In Proceedings of the International Symposium on Memory Management. 84-95. DOI: http://dx.doi.org/10.1145/3210563.3210571

[3] Dong Chen, Fangzhou Liu, Chen Ding, and Sreepathi Pai. 2018. Locality analysis through static parallel sampling. In Proceedings of the ACM SIGPLAN Conference on Programming Language Design and Implementation. 557-570. DOI : http://dx.doi.org/10.1145/3192366.3192402

[4] David Eklov and Erik Hagersten. 2010. StatStack: Efficient modeling of LRU caches. In Proceedings of the IEEE International Symposium on Performance Analysis of Systems and Software. 55-65.

[5] Christos Faloutsos, Yossi Matias, and Abraham Silberschatz. 1996. Modeling Skewed Distribution Using Multifractals and the '80-20' Law. In Proceedings ofInternational Conference on Very Large Data Bases. 307-317. http://www.vldb.org/conf/1996/P307.PDF

[6] Xiameng Hu, Xiaolin Wang, Lan Zhou, Yingwei Luo, Chen Ding, and Zhenlin Wang. 2016. Kinetic Modeling of Data Eviction in Cache. In Proceedings of USENIX Annual Technical Conference. 351364. https://www.usenix.org/conference/atc16/technical-sessions/ presentation/hu

[7] Yannis E. Ioannidis and Viswanath Poosala. 1995. Balancing Histogram Optimality and Practicality for Query Result Size Estimation. In Proceedings of the ACM SIGMOD International Conference on Management of Data. 233-244. DOI : http://dx.doi.org/10.1145/223784.223841

[8] M. K. Qureshi and Y. N. Patt. 2006. Utility-Based Cache Partitioning: A Low-Overhead, High-Performance, Runtime Mechanism to Partition Shared Caches. In Proceedings of the International Symposium on Microarchitecture.

[9] Manfred R. Schroeder. 1991. Fractals, chaos, power laws - minutes from an infinite paradise. Freeman. http://www.worldcat.org/oclc/21522909

[10] David K. Tam, Reza Azimi, Livio Soares, and Michael Stumm. 2009. RapidMRC: approximating L2 miss rate curves on commodity systems for online optimizations. In Proceedings of the International Conference on Architectural Support for Programming Languages and Operating Systems. 121-132.

[11] Carl A. Waldspurger, Trausti Saemundsson, Irfan Ahmad, and Nohhyun Park. 2017. Cache Modeling and Optimization using Miniature Simulations. In Proceedings of USENIX Annual Technical Conference. 487-498. https://www.usenix.org/conference/atc17/ technical-sessions/presentation/waldspurger

[12] Wang et al. 2015. Optimal Program Symbiosis in Shared Cache. In Proceedings of the IEEE International Symposium on Cluster Computing and the Grid.

[13] Xiaoya Xiang, Chen Ding, Hao Luo, and Bin Bao. 2013. HOTL: a higher order theory of locality. In Proceedings of the International Conference on Architectural Support for Programming Languages and Operating Systems. 343-356.

[14] Yaocheng Xiang, Xiaolin Wang, Zihui Huang, Zeyu Wang, Yingwei Luo, and Zhenlin Wang. 2018. DCAPS: dynamic cache allocation with partial sharing. In Proceedings of the EuroSys Conference. 13:1-13:15. DOI : http://dx.doi.org/10.1145/3190508.3190511 